\title{
Long term complications of inferior vena cava thrombosis
}

\author{
M Häusler, D Hübner, T Delhaas, E G Mühler
}

\begin{abstract}
Aim-To evaluate the long term outcome after paediatric inferior vena cava (IVC) thrombosis.

Methods-A combined retrospective and prospective study on infants and children with IVC thrombosis treated at Aachen and Maastricht University Hospitals between 1980 and 1999.

Results-Forty patients were enrolled, including four with preceding cardiac catheterisation, 18 with central venous saphenous lines, and an additional eight with umbilical venous catheters. Six patients died within three months of diagnosis; one patient was lost to follow up. Twelve of the remaining 33 patients had suffered from limited IVC thrombosis; during follow up (for up to nine years) none showed persisting caval obstruction (successful thrombolysis, $\mathbf{n}=2$; spontaneous recanalisation, $n=10$ ). The remaining 21 patients presented with extensive IVC thrombosis. During follow up (for up to 18 years) complete restitution was found in only four cases (one thrombolysis, two surgery, one spontaneous recanalisation). Persisting iliac and/or caval venous obstruction occurred in 17 patients, including six with thrombolysis. Varicose veins were found in 12, and postthrombotic syndrome in seven of these cases. According to Kaplan-Meier analysis, $30 \%$ of patients with persisting venous disease will develop post-thrombotic syndrome within 10 years of the thrombotic event.

Conclusions-Infants and children with extensive IVC thrombosis are at high risk for persisting venous disease and serious long term complications. Prospective trials are urgently needed to establish effective treatment strategies and to improve long term prognosis. Central venous catheters, contributing to IVC thrombosis in the majority of cases reported here, should be inserted only if essential.

(Arch Dis Child 2001;85:228-233)
\end{abstract}

Keywords: vena cava; thrombosis; post-thrombotic syndrome; catheterisation; central venous; nephroblastoma

Department of Paediatrics, University

Hospital Maastricht, Netherlands

T Delhaas

Correspondence to:

Dr Häusler

Haeusler@RWTH-Aachen.de

Accepted 29 May 2001 only four patients had survived. Since 1960 hereditary thrombophilia, nephrotic syndrome,
The first extensive review on paediatric inferior vena cava (IVC) thrombosis was published in 1960, when Kaufman analysed 21 cases published since $1909 .{ }^{1}$ Most of these were sec- antiphospholipid autoantibodies, and abdominal trauma have been identified as further risk factors. $^{2-7}$ While medical progress has improved survival prospects, modern therapeutic modalities may even have increased the prevalence of IVC thrombosis: insertion of central venous catheters (CVC) or cardiac catheters via femoral access may cause complete IVC occlusion in up to $14.5 \%$ of young infants. ${ }^{8}$ The incidence of CVC related paediatric deep venous thrombosis in general has been calculated to be at least 3.5 per 10000 hospital admissions, and $10 \%$ of these thromboses may affect the IVC. ${ }^{10}$ Therefore, a considerable number of patients can be assumed at the present time to have suffered from paediatric IVC thrombosis. Single case reports on adults and children suggest a serious risk of these patients developing complications such as Budd-Chiari syndrome, renal venous thrombosis, thrombosis of collaterals, bleeding from gastrointestinal varices, spinal claudication, or venous embolism. ${ }^{21-15}$ Data from the Canadian Childhood Thrombophilia Registry have revealed a $12.4 \%$ rate of post-thrombotic syndrome in a heterogeneous group of patients with central venous thromboses after a mean follow up of three years. This study has also shown that patients with lower venous system thrombosis may have the highest risk of developing post-thrombotic syndrome. ${ }^{16}$ During follow up of adults with IVC thrombosis, leg swelling was reported in $91 \%$, deep venous thrombosis in $71 \%$, venous ulcers in $54 \%$, and venous claudication in $20 \%$ of cases. ${ }^{17}$ However, despite these important sequelae in adults, the long term outcome after paediatric IVC thrombosis is unknown. This combined prospective and retrospective study is the first to describe the long term outcome in a larger series of 40 patients presenting with IVC thrombosis between birth and 13 years of age.

\section{Patients and methods}

PATIENTS

Forty patients with IVC thrombosis, diagnosed between 1980 and 1999 at the Departments of Paediatrics, Paediatric Cardiology, and Clinical Radiology of Aachen University Hospital (Germany) or at the Department of Paediatrics of Maastricht University Hospital (Netherlands) were included. Twenty one patients were neonates (table 1); six were suffering from cyanotic, and four from acyanotic heart disease. Thirty three cases were identified in 1997 during a retrospective database search; first outcome data of 12 of these patients, who presented with IVC thrombosis between birth and 4 months of age at Aachen University Hospital and who were reinvestigated in 1997, 
Table 1 Clinical data for 40 patients with inferior vena cava thrombosis

\begin{tabular}{lrllll}
\hline Age at onset & $n$ & $\begin{array}{l}\text { Central venous } \\
\text { saphenous catheter }\end{array}$ & $\begin{array}{l}\text { Preceding cardiac } \\
\text { catheterisation }\end{array}$ & $\begin{array}{l}\text { Umbilical venous } \\
\text { catheter }\end{array}$ & Nephroblastoma \\
\hline Neonatal period (premature infants) & 12 & 4 & 1 & 3 & \\
Neonatal period (term infants) & 9 & 3 & 1 & 3 & 1 \\
2 to 12 months & 14 & 10 & 2 & & 1 \\
1 to 5 years & 3 & & & & \\
7 to 13 years & 2 & 1 & & \\
\hline
\end{tabular}

have been published previously. ${ }^{4}$ Seven additional patients presented with IVC thrombosis between 1997 and 1999.

DIAGNOSIS AND EXTENT OF IVC THROMBOSIS

IVC thrombosis was diagnosed in 36 patients at the time of manifestation (median age at diagnosis 3 weeks; range 1 day to 13 years), and in four patients when puncture of femoral veins showed venous obstruction during cardiac catheterisation at a later age (median 8 months; range 6 months to 3 years). In the latter cases the thrombosis was considered to have occurred at the date of a preceding insertion of a CVC $(n=2)$ or cardiac catheter $(n=2)$. In all patients ultrasound was the method chosen to confirm the diagnosis of IVC thrombosis. Additional cavography was performed in four, and computed tomography in two patients.

According to imaging data, two patient groups with limited and with extensive thrombosis could be distinguished. Limited thrombosis was present in 16 patients. In 10 of these patients the thrombosis extended into the right atrium, originating either from the umbilical vein in patients with umbilical CVC $(8 / 10)$ or from the hepatic portion of the IVC in patients with saphenous CVC (2/10). None were suffering from renal venous thrombosis or adrenal bleeding. In the remaining six cases the thrombosis was restricted either to the pararenal $(n=5)$ or to the intrahepatic segment $(n=1)$. In three of these cases it was related to a CVC; in the others it was associated with neonatal renal venous thrombosis.

Extensive thrombosis occurred in 24 patients and was defined as extending to at least two caval segments (intrahepatic, pararenal, or infrarenal) or to the infrarenal IVC and iliac veins. This group comprised 12 patients with a CVC, six with spontaneous postnatal IVC thrombosis, four who had undergone cardiac catheterisation, and two with nephroblastomas. Four of these patients presented with additional renal venous thrombosis, and a further five patients with renal venous thrombosis and adrenal bleeding.

EARLY MANAGEMENT

Thrombolysis was performed in $13 / 40$ patients, using urokinase $(\mathrm{n}=10)$, streptokinase $(\mathrm{n}=1)$, or tissue type plasminogen activator $(\mathrm{n}=2)$. Because different physicians had diagnosed the patients over an extended period of time, no uniform treatment protocol or inclusion criteria for thrombolysis were used. Surgical thrombectomy was performed in one premature infant with infectious thrombosis and in the two patients with nephroblastoma. In one of the latter the pararenal caval wall was replaced by a vascular prosthesis; the obstruction of the stenotic infrarenal IVC lumen could not be rectified.

EARLY MORTALITY

Six patients, three with limited (19\%) and three with extensive $(12.5 \%)$ thrombosis, died within three months of diagnosis. In none of these cases was the IVC thrombosis itself or its therapy the only cause of death. These infants died from multiorgan failure $(n=1)$, pulmonary hypoplasia $(n=1)$, septicaemia $(n=1)$, superior vena cava syndrome after successful arterial switch surgery $(n=1)$, and heart failure in congenital heart disease $(n=2)$. An autopsy performed in three of these infants ruled out pulmonary embolism in all cases.

\section{FOLLOW UP INVESTIGATIONS}

Early follow up (three months) was available from all patients in whom IVC thrombosis had been diagnosed at the time of manifestation $(n=36)$. Prospective reinvestigations initiated in 1997 were aimed at including all surviving patients with extensive thrombosis and patients for whom retrospective data analysis provided no clear cut confirmation of complete venous recanalisation. Finally, all 21 surviving patients with extensive thrombosis and five of the 13 surviving patients with limited thrombosis could be reinvestigated prospectively. Only one patient with limited thrombosis and uncertain outcome was completely lost to follow up. The investigation protocol included a detailed history (for example, leg or abdominal pain), clinical investigations concerning varicose veins and symptoms of post-thrombotic syndrome (ulcers, oedema, exanthema), and sonography of abdominal, iliac, and femoral veins. Serial prospective follow up investigations were and still are conducted in all patients with persisting occlusion or obstruction of the IVC and/or the iliac veins. Depending on the clinical and sonographic findings, additional contrast venography $(n=12)$, computed tomography $(n=6)$, or magnetic resonance imaging $(n=4)$ of abdominal and iliac veins was performed in 15 patients, including all but three of the long term survivors with persisting venous disease. To rule out pulmonary artery hypertension indicative of chronic pulmonary embolism, echocardiographic and electrocardiographic examinations were performed in all patients with persisting IVC thrombosis and/or iliac venous disease.

Laboratory data concerning hereditary thrombophilic risk factors, for example, protein $\mathrm{C}$ and $\mathrm{S}$ deficiency, homocystein, antithrombin, cholesterol, triglyceride, and lipoprotein (a) concentrations, thrombin time, 


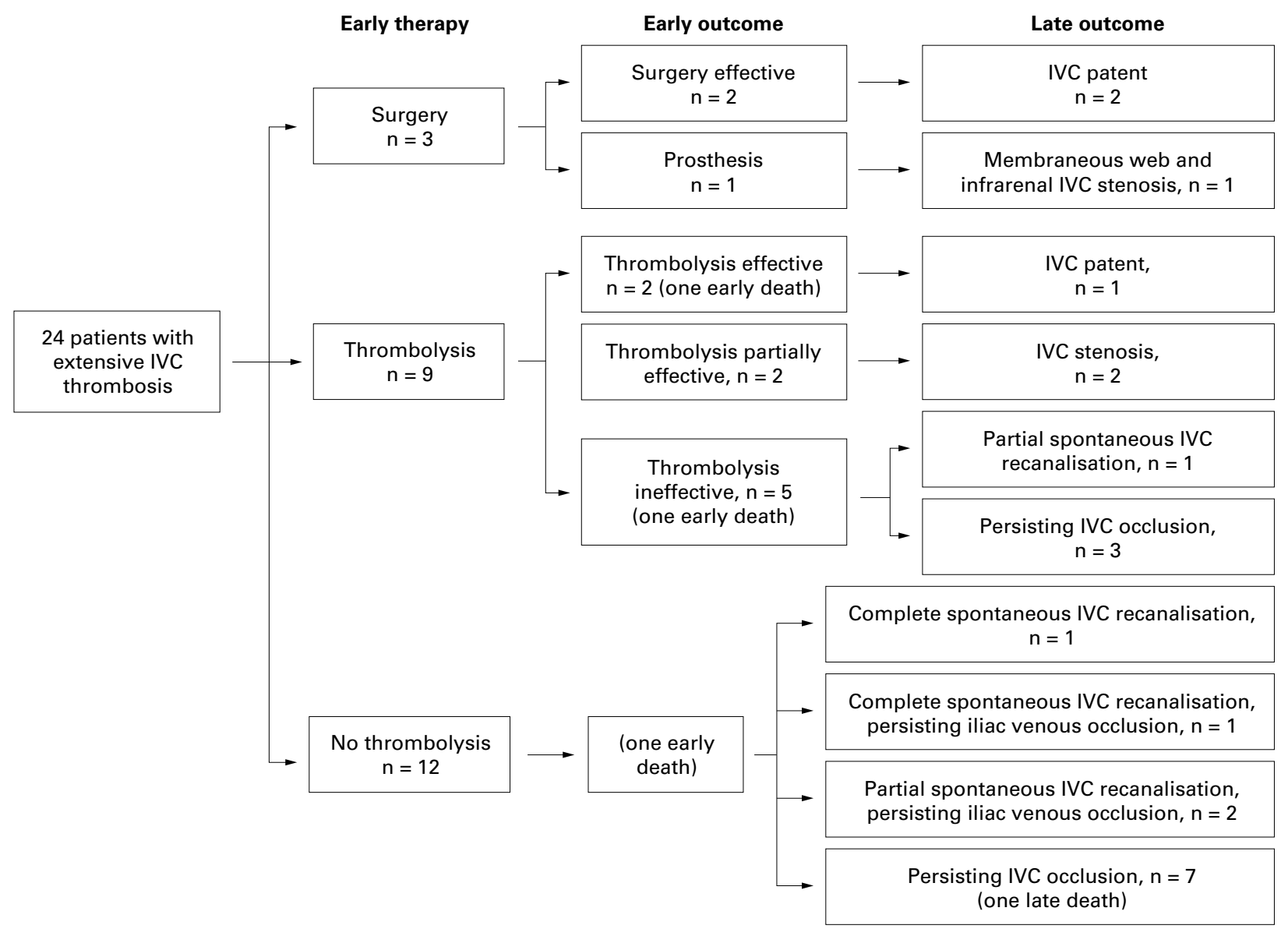

Figure 1 Clinical course of 24 patients with extensive inferior vena cava (IVC) thrombosis.

resistance to activated protein $\mathrm{C}$, and plasminogen activity, were obtained from 22 patients. In cases with a low activated protein $\mathrm{C}$ ratio, resistance to activated protein $\mathrm{C}$ was confirmed by documentation of the factor $\mathrm{V}$ Leiden mutation. Disease free survival time was calculated according to the Kaplan-Meier formula.

\section{Results}

LATE MORTALITY

Late sudden death occurred in one 18 year old female with cyanotic heart disease, who was on oral anticoagulation after recurrent phlebothrombosis. While putting on her compression stockings, she suddenly lost consciousness and died. Although no autopsy was performed, the clinical circumstances, the absence of cardiac arrhythmia during her lifetime, and the fact that she was non-compliant for her oral anticoagulation (international normalised ratio shortly before death: 1.2) strongly suggest fatal thromboembolism.

OUTCOME AFTER LIMITED THROMBOSIS

Late follow up data were available from seven of the 10 patients with IVC thrombosis extending into the right atrium; two of these patients had died early and one patient was lost to follow up. Although these seven patients received neither thrombolysis nor heparin therapy, spontaneous and complete disappearance of the thrombus was observed in six of them between two months and two years after thrombosis (median follow up 10 months, range 5 months to 2 years). The seventh patient (actual follow up 15 months) has hitherto shown a notable spontaneous reduction of the thrombus size, with a small non-obstructive remnant.

Five of the remaining six patients with limited thrombosis not extending into the right atrium survived the early follow up period (median follow up 6 years, range 9 months to 8 years 9 months). In two of them urokinase thrombolysis had effectively resolved thrombosis of the pararenal IVC. In two further patients in whom IVC thrombosis originated from the tip of a saphenous CVC, heparin therapy $\left(10000 \mathrm{U} / \mathrm{m}^{2} /\right.$ day) had resulted in complete disappearance of the thrombus within 24 hours. In the last patient, in whom a pararenal IVC thrombosis originated from thrombotic plugs occluding both renal veins, heparin therapy had resulted in complete recanalisation of the IVC within seven days.

OUTCOME AFTER EXTENSIVE THROMBOSIS

During prospective reinvestigations (median follow up 10 years 3 months, range 3 months to 18 years 2 months), complete patency of the IVC and of iliac veins was found in only four of the surviving 21 patients with extensive thrombosis (effective urokinase thrombolysis, $n=1$; surgical thrombectomy, $\mathrm{n}=2$; complete spontaneous recanalisation, $\mathrm{n}=1$ ). Despite 


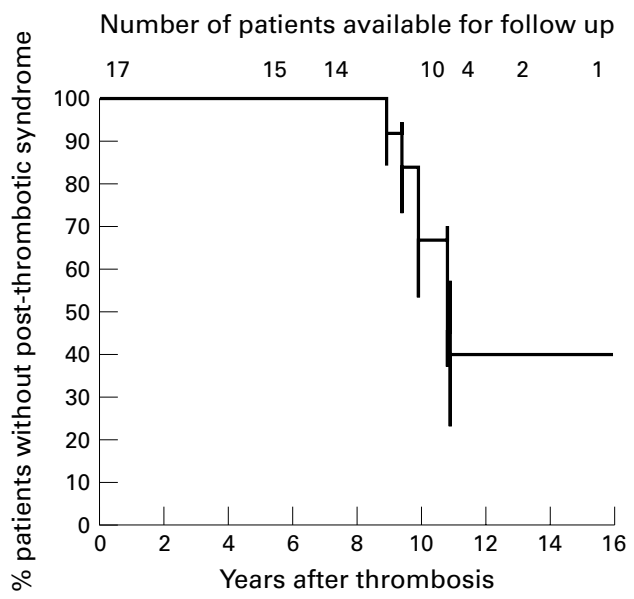

Figure 2 Survival time without post-thrombotic syndrome, calculated from follow up data of 17 patients with persisting venous pathology. Vertical bars indicate the standard error.

thrombolytic treatment $(\mathrm{n}=6)$ or surgery $(n=1)$, persisting caval occlusion, IVC stenosis, and/or occlusion of iliac veins was found in the remaining 17 patients (fig 1 ), including the four patients with late diagnosis during cardiac catheterisation. Ten of these 17 patients suffer from exercise dependent or nocturnal leg pain and two from abdominal pain. The prevalence and extent of varicose veins found in 12 patients (two of four patients with a follow up period of up to 8 years, and 10 of 13 patients with a follow up of more than 8 years) were found to increase with age. During serial follow up investigations of the 17 patients with persisting venous disease, seven patients have hitherto developed post-thrombotic syndrome, presenting with leg oedema $(n=5)$, leg exanthema $(n=2)$, phlebothrombosis of the lower limbs $(n=1)$, or incipient leg ulcer $(n=1)$. Kaplan-Meier analysis shows that $30 \%$ of patients with persisting venous disease will develop post-thrombotic syndrome (exanthema, ulcers, oedema, thrombotic complications; varicose veins excluded) within 10 years of IVC thrombosis (fig 2).

In all nine cases with persisting caval disease where contrast enhanced computed tomography and/or magnetic resonance imaging of the abdomen were performed, lumbar, mesenterial, and spinal collaterals were found. No patient has hitherto presented with spinal claudication or with bleeding from gastrointestinal varices.

An intracaval membranous web was found in three patients. In an 11 year old girl it was situated at the proximal lineage of the persisting IVC occlusion and was considered to reflect incomplete IVC recanalisation. An intrahepatic web was found in a 9 year old boy with heterozygous factor $\mathrm{V}$ Leiden mutation (fig 3). Because this patient showed complete spontaneous recanalisation of the distal IVC, the web may be caused by incomplete recanalisation of the hepatic IVC. The 12 year old boy with an IVC prosthesis was also found to have an intrahepatic web. Oral anticoagulation had been performed for six months after surgery. As sonography had shown normal findings until four years after thrombectomy, the web can only be attributed to asymptomatic partial rethrombosis.

None of the patients with extensive thrombosis has shown echo- or electrocardiographic signs of pulmonary artery hypertension.

HEREDITARY THROMBOPHILIA

Laboratory testing for hereditary thrombophilia revealed positive findings in five of the 22 investigated patients (table 2). In the patient with heterozygous protein $S$ type 1 deficiency (maternal inheritance), heparin therapy effectively resolved limited IVC thrombosis, as did
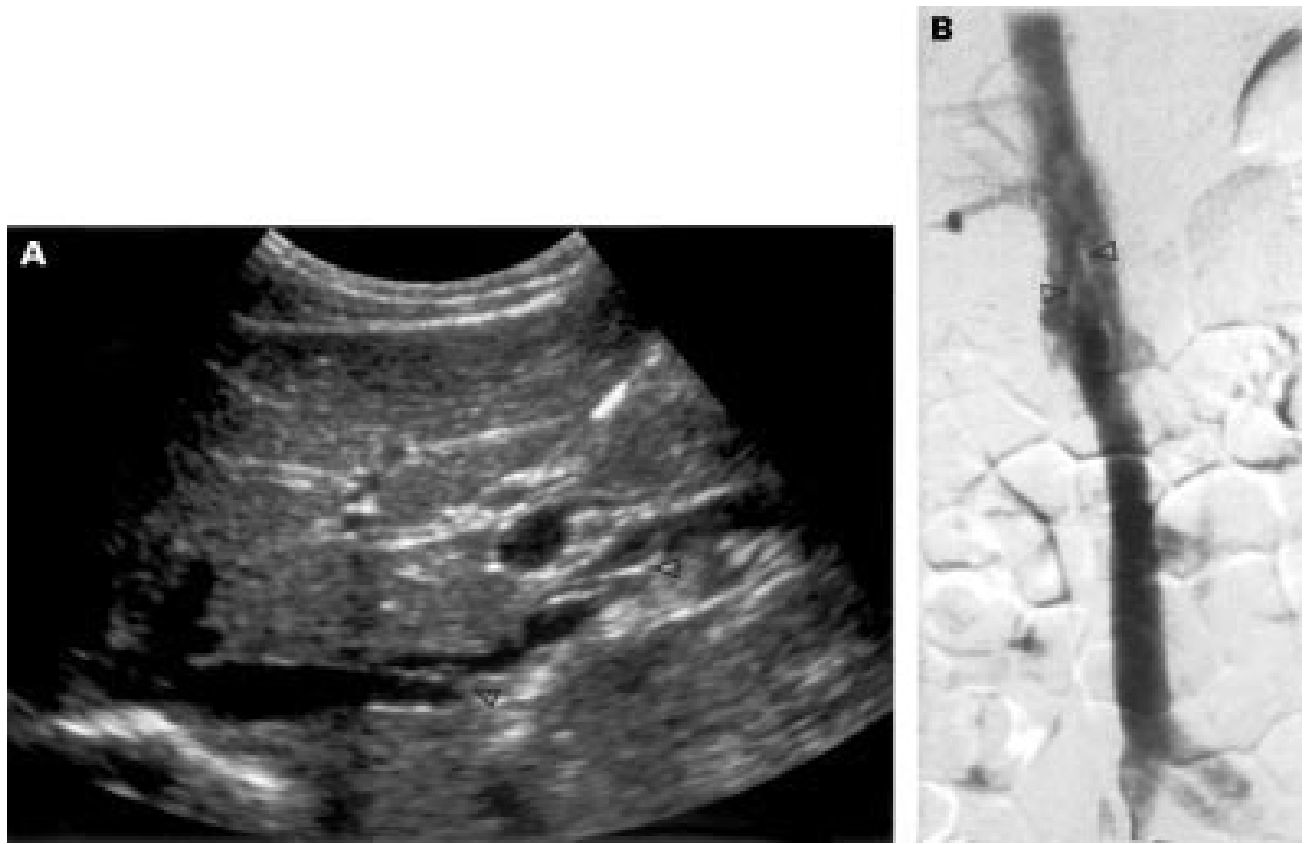

Figure 3 Sagittal ultrasound image of the inferior vena cava $(A)$ and cavography after transfemoral injection of contrast media (B), showing intraluminal and wall adherent web like septa (arrows) within the proximal inferior vena cava of a 9 year old boy. 
Table 2 Prevalence of hereditary thrombophilia and outcome in 22 patients with inferior vena cava thrombosis

\begin{tabular}{lll}
\hline & Outcome & \\
\cline { 2 - 3 } & Complete recanalisation & Persisting venous pathology \\
\hline $\begin{array}{c}\text { Limited IVC thrombosis }(\mathrm{n}=5) \\
\text { (including 2 patients with CVC) }\end{array}$ & $\begin{array}{l}\mathrm{n}=5 \\
(\mathrm{hPS} 1, \mathrm{n}=1 ; \mathrm{hFV}, \mathrm{n}=1)\end{array}$ & $\mathrm{n}=0$ \\
$\begin{array}{c}\text { Extensive IVC thrombosis ( } \mathrm{n}=17) \\
\text { (including 10 patients with CVC) }\end{array}$ & $\mathrm{n}=3$ & $\begin{array}{l}\mathrm{n}=14 \\
(\mathrm{hFV}, \mathrm{n}=3)\end{array}$ \\
\hline
\end{tabular}

$\overline{\mathrm{CVC} \text {, central venous catheter; } \mathrm{hPS} 1 \text {, heterozygous protein } \mathrm{S} \text { type } 1 \text { deficiency; } \mathrm{hFV} \text {, heterozygous }}$ factor V Leiden mutation.

urokinase thrombolysis in the patient with heterozygous factor $\mathrm{V}$ Leiden deficiency. Urokinase thrombolysis had also been performed in two of the three patients with extensive IVC thrombosis and heterozgyous factor $\mathrm{V}$ Leiden deficiency. It had been ineffective in one patient and partially effective in the other.

ORAL ANTICOAGULATION

Immediately after the diagnosis of IVC thrombosis, oral anticoagulation was performed only in the patient with a caval prosthesis. Long term oral anticoagulation was performed in two girls with congenital heart disease and persisting IVC occlusion: in one patient who underwent Fontan type surgery but did not suffer from thrombotic complications, and in one who had suffered acute phlebothrombosis at the age of 15 years and later died from an assumed lethal thromboembolic event. All other patients were without medical prophylaxis, and none has suffered so far from a symptomatic second thrombotic or thromboembolic event.

\section{Discussion}

Long term complications of IVC thrombosis, which are well known in adults, can be assumed for paediatric patients on the basis of single case reports and are strongly suggested by the results of the Canadian Childhood Thrombophilia Registry; they have not, however, been specified in detail in childhood. ${ }^{21-17}$ This is the first study to focus selectively on the long term outcome after paediatric IVC thrombosis. It shows that the prognosis after IVC thrombosis may depend predominantly on its extent: persisting venous occlusion was not found after limited thrombosis, but was found in $80 \%$ of cases after extensive thrombosis. In these patients with persisting venous disease, leg pain and abdominal pain were frequent. Varicose veins occurred from a young age and increased with time, and postthrombotic syndrome was present in $30 \%$ of the patients at the 10 year follow up. This long follow up period and the focus on patients with persisting venous disease may account for the high prevalence of post-thrombotic syndrome, which would have been even higher if, as in the Canadian registry, we had also included patients with isolated leg pain. Some patients showed residual IVC stenosis or septation, or developed an intravascular membranous web (fig 3), changes that predispose to sudden rethrombosis of the IVC, Budd-Chiari syndrome, or peripheral venous thrombosis. ${ }^{2} 1819$

Although these findings show that intensive efforts aimed at restoring vessel patency in cases of acute extensive thrombosis and at preventing late complications may be justified, specific treatment strategies are not available for acute or chronic venous obstruction and can only be adapted from general recommendations (for detailed reviews see Michelson and colleagues $^{20}$ and Verstraete and colleagues ${ }^{21}$ ). According to these recommendations, anticoagulation therapy should be performed for at least three months in children aged more than 2 months who are suffering from extensive venous thrombosis. This should also be considered in neonates. Thrombolysis, in contrast, remains highly individualised.

In our patients the efficacy of thrombolysis seemed to depend predominantly on the extent of the thrombosis, being effective in two of four patients with limited and in two of nine patients with extensive thrombosis (fig 1). Heparin therapy, performed in three patients with limited thrombosis, was effective in all cases. However, as this was not a prospective randomised trial, our data should not lead to thrombolytic therapy being withheld from patients with limited thrombosis. We performed oral anticoagulation less frequently than recommended after manifestation of IVC thrombosis, and we did not perform routine long term anticoagulation. Nevertheless, no second symptomatic thrombotic or thromboembolic events were observed within 10 years of IVC thrombosis, which might suggest that general long term anticoagulation is not required for children with persisting venous disease. However, we cannot rule out the possibility that spontaneous recanalisation might have been more frequent if early anticoagulation had been performed in more cases. After discontinuation of oral anticoagulation, the patient with a caval prosthesis developed an asymptomatic intracaval membranous web (fig 3 ), and 13 years after IVC thrombosis one patient died from an assumed thromboembolic complication. Additionally, the presence of heterozygous protein $\mathrm{S}(4.5 \%)$ or factor V Leiden deficiency (18\%) (table 2)-the latter is known to be more frequent in patients with IVC thrombosis compared to the normal population (5\%) - may be important not only to the occurrence of the thrombosis. ${ }^{3}$ After adolescence, patients with hereditary thrombophilia and persistent venous disease will have an increased risk of rethrombosis and may then require long term anticoagulation. ${ }^{22}$

The question of which follow up examinations should be performed is another difficult matter. Phlebologic methods established in adults have not yet been standardised for paediatric patients. ${ }^{23}$ Clinical investigations are limited by physiological changes related to growth of the extremities and to skin thickness, and the accuracy of sonography, especially for evaluation of the infrarenal and pelvic veins, is unknown for paediatric patients. Even the optimal diagnostic approach to acute deep venous thrombosis in adults is still a matter of controversial discussion. Though less invasive, B mode or colour duplex ultrasound do not achieve $100 \%$ sensitivity or specificity for detection of venous obstruction compared with 
venography. ${ }^{24} 25$ Magnetic resonance imaging or computed tomography-promising techniques for evaluation of intrapelvic pathology $y^{24}$ - still require technical optimisation. Our experience suggests that, while sonography may be useful in revealing the venous morphology, it is insufficiently reliable for assessment of the functional impact of morphologic findings. In one patient, for example, sonography suggested narrowing of the infrahepatic IVC lumen without blood flow obstruction, whereas venography showed that most blood was being drained from the legs to paravertebral and azygous collaterals.

In all patients with acute IVC thrombosis we now intend to perform thrombolysis, using urokinase or tissue type plasminogen activator, depending on the clinical experience of the respective hospital. This will be followed, in accordance with the above mentioned guidelines, by oral anticoagulation. ${ }^{20}{ }^{21}$ In patients who have recovered from acute thrombosis and in all patients with late diagnosis, we now perform a detailed investigation of the venous system. This includes clinical and sonographic examinations in all cases and, depending on these findings, phlebography of the legs in order to confirm deep venous thrombosis, venography of iliac collaterals to detect the functional impact of vessel obstruction, and computed tomography or magnetic resonance imaging for detailed presentation of further pelvic, lumbar, mesenterial, or spinal collaterals. On the one hand, these investigations help us to decide whether regular follow up investigations are required; on the other hand, they have already been shown to be helpful in ruling out new thromboses in patients presenting with acute clinical symptoms during follow up. Routine clinical and sonographic follow up investigations are performed at 12 month intervals. For all patients with persisting venous disease we recommend knee length compression stockings, an established treatment modality of post-thrombotic syndrome in adults which has already reversed peripheral leg oedema in two of our patients, suggesting some benefit for paediatric patients as well. ${ }^{27}$

Although we have observed serious sequelae of IVC thrombosis in a considerable number of patients, the present study probably underestimates the problems associated with paediatric IVC thrombosis. Our patients with persisting venous disease now are at a median age of 11 years (range 6 months to 19 years) and during later life their post-thrombotic sequelae may well deteriorate even further. The widespread use of CVCs, the most important risk factor among our patients, additionally suggests that IVC thrombosis is very common. Moreover, bearing in mind those patients who were diagnosed late after the thrombotic event, many patients can be assumed to be actually suffering from hitherto unrecognised persisting IVC occlusion.

These data strongly recommend preventing the occurrence of IVC thrombosis in infants and children; restricting the insertion of CVCs to as few patients as possible may be the most important measure. The addition of heparin to the infundate or anticoagulation of patients with CVCs may additionally reduce the risk of vessel occlusion. ${ }^{28}$ These modalities, however, need further evaluation and optimisation for paediatric patients. To improve the prognosis of children with caval venous occlusion, prospective trials on diagnostic modalities and therapeutic management of acute and chronic IVC thrombosis are urgently needed.

1 Kaufman S. Obstruction of the inferior vena cava in childhood. Pediatrics 1960;19:852-8.

2 Halbmayer WM, Radek J, Duschet P, et al. Recurrent venous thromboses in hypoplasia of the vena cava inferior and factor XII deficiency. Dtsch Med Wochenschr 1993;118: 1561-6.

3 Münchow N, Kosch A, Schobess R, et al. Role of genetic prothrombotic risk factors in childhood cava vein thrombosis. Eur F Pediatr 1999;158(suppl 3):S109-12.

4 Häusler M, Duque D, Merz U, et al. The clinical outcome after inferior vena cava thrombosis in early infancy. Eur $\mathcal{F}$ Pediatr 1999;158:416-20.

5 Alexander F, Campbell WA. Congenital nephrotic syndrome and renal vein thrombosis in infancy. $\mathcal{F}$ Clin Pathol $1971 ; 24: 27-40$

6 Contractor S, Hiatt M, Kosmin M, et al. Neonatal thrombosis with anticardiolipin antibody in baby and mother. Am $\mathcal{F}$ Perinatol 1992;9:409-10.

7 Redel D, Fehske W, Kirchhoff PG. Budd-Chiari syndrome in a child with post-traumatic obstruction of the inferior vena cava Radiology 1981;139:151-4.

8 Laurin S, Lundström NR. Venous thrombosis after cardiac catheterization in infants. Acta Radiol 1987;28:241-6.

9 Pippus KG, Giacomantonio JM, Gillis DA, et al. Thrombotic complications of saphenous central venous lines. $\mathcal{f}$ Pediatr Surg 1994;29:1218-19.

10 Massicotte MP, Dix D, Monagle P, et al. Central venous catheter-related thrombosis in children: analysis of the Canadian Registry of Venous Thromboembolic Complications. F Pediatr 1998;133:770-6.

11 Taneja A, Mitra SK, Moghe PD, et al. Budd-Chiari syndrome in childhood secondary to inferior vena caval obstruction. Pediatrics 1979;63:808-12.

12 Lash C, Radhakrishnan J, McFadden JC. Renal vein thrombosis secondary to absent inferior vena cava. Urology 1998; $51: 829-30$.

13 Zhou H, Janssen D, Günther E, et al. Fatal bleeding from duodenal varices as a late complication of neonatal thrombosis of the inferior vena cava. Virchows Arch A Pathol Anat bosis of the inferior vena cava.
Histopathol 1992;420:367-70.

14 Blättler W, Krayenbuhl C. Spinal canal stenosis syndrome by venous collateralization of an inferior cava thrombosis. Phlebologie 1993;46:411-14.

15 Coupland GA, Reeve TS. The sequelae of inferior vena caval interruption. Aust N Z F Surg 1975;45:245-51.

16 Monagle $\mathrm{P}$, Adams M, Mahoney M, et al. Outcome of pediatric thromboembolic disease: a report from the Canadian Childhood Thrombophilia Registry. Pediatr Res 2000;47: 763-6.

17 Jackson BT, Thomas ML. Post-thrombotic inferior vena caval obstruction. A review of 24 patients. BMF 1970;1:1822 .

18 Blanshard C, Dodge G, Pasi J, et al. Membranous obstruction of the inferior vena cava in a patient with factor $\mathrm{V}$ Leiden: evidence for a post-thrombotic aetiology. $\mathcal{F}$ Hepatol Leiden: evidence

19 Arao $M$, Ogura $H$, Ino $T$, et al. Unusual inferior vena cava obstruction causing extensive thrombosis: an intravascular endoscopic observation. Intern Med 1993;32:861-4.

20 Michelson AD, Bovill E, Monagle P, et al. Antithrombotic therapy in children. Chest 1998;114(suppl):748S-69S.

21 Verstraete $M$, Prentice CRM, Samama M, et al. European view on the North American Fith Consensus on Antithrombotic Therapy. Chest 2000;117:1755-70.

22 Pabinger I, Schneider B. Thrombotic risk in hereditary antithrombin III, protein C, or protein S deficiency. A cooperative, retrospective study. Gesellschaft für Thrombose- und Hämostaseforschung (GTH) Study Group on Natural Inhibitors. Arterioscler Thromb Vasc Biol 1996;16:742-8.

23 Perrin M, Guex JJ. Edema and leg volume: methods of assessment. Angiology 2000;51:9-12

24 Tapson VF, Carroll BA, Davidson BL, et al. The diagnostic approach to acute venous thromboembolism. Am $\mathcal{F}$ Respir Crit Care Med 1999;160:1043-66.

25 Davidson BL, Elliott CG, Lensing AWA. Low accuracy of color doppler ultrasound in the detection of proximal leg vein thrombosis in asymptomatic high-risk patients. Ann Intern Med 1992;117:735-8.

26 Baldt MM, Zontsich T, Stumpflen A, et al. Deep venous thrombosis of the lower extremity: efficacy of spiral CT venography compared with convention diagnosis. Radiology 1996;200:423-8.

27 Clement DL. Management of venous edema: insights from an international task force. Angiology 2000;51:13-17.

28 Hentschel R, Wiescholek J, von Lengerke J, et al. Coagulation-associated complications of indwelling arteCoagulation-associated complications of indwelling arte-
rial and central venous catheters during heparin prophylaxis. Eur f Pediatr 1999;158(suppl 3):S126-9. 